Service social

\title{
Traduction et validation d'un instrument de mesure de la cohésion et de l'adaptabilité des couples et des familles
}

\section{Claire Dumont}

Volume 55, numéro 1, 2009

URI : https://id.erudit.org/iderudit/029488ar

DOI : https://doi.org/10.7202/029488ar

Aller au sommaire du numéro

Éditeur(s)

École de service social de l'Université Laval

ISSN

1708-1734 (numérique)

Découvrir la revue

Citer cet article

Dumont, C. (2009). Traduction et validation d'un instrument de mesure de la cohésion et de l'adaptabilité des couples et des familles. Service social, 55(1),

31-46. https://doi.org/10.7202/029488ar
Résumé de l'article

Un instrument de mesure de la cohésion et de l'adaptabilité des couples et des familles a été traduit en français et utilisé dans une étude visant à documenter la contribution de la famille au processus de réadaptation des adultes ayant subi un traumatisme craniocérébral. L'instrument a été administré à deux échantillons dans le but d'évaluer la cohérence interne et la validité de construit (respectivement 27 et 53 personnes). La réadaptation visant principalement l'amélioration de la participation sociale, des analyses corrélationnelles avec une mesure de la participation sociale ont également été effectuées avec le deuxième échantillon. Les résultats démontrent que la traduction a des propriétés métrologiques se rapprochant de la version initiale anglaise. La contribution de la famille pour la participation sociale s'est avérée être significative, mais relativement modeste. La nature des variables et l'échelle utilisée peuvent en partie expliquer ce résultat. 


\section{Traduction et validation d'un instrument de mesure de la cohésion et de l'adaptabilité des couples et des familles}

Claire DUMONT

Doctorat en santé communautaire

Conseillère en évaluation de programmes

Institut de réadaptation en déficience physique de Québec

Un instrument de mesure de la cohésion et de l'adaptabilité des couples et des familles a été traduit en français et utilisé dans une étude visant à documenter la contribution de la famille au processus de réadaptation des adultes ayant subi un traumatisme craniocérébral. L'instrument a été administré à deux échantillons dans le but d’évaluer la cohérence interne et la validité de construit (respectivement 27 et 53 personnes). La réadaptation visant principalement l'amélioration de la participation sociale, des analyses corrélationnelles avec une mesure de la participation sociale ont également été effectuées avec le deuxième échantillon. Les résultats démontrent que la traduction a des propriétés métrologiques se rapprochant de la version initiale anglaise. La contribution de la famille pour la participation sociale s'est avérée être significative, mais relativement modeste. La nature des variables et l'échelle utilisée peuvent en partie expliquer ce résultat.

Mots-clés : cohésion des familles, adaptabilité des familles, participation sociale, traumatisme craniocérébral, réadaptation.

An instrument measuring family and couple cohesion and adaptability was translated into French and used in a study aiming at documenting the contribution of the family in the rehabilitation process of adults having sustained a traumatic brain-injury. The instrument was administered to two samples in order of estimating internal consistency and construct validity (respectively 27 and 53 persons). As rehabilitation aims mainly at improving social participation, corelational analyses with a measure of social participation were also performed with the second sample. The results demonstrate that the translation has psychometric properties getting closer to the English initial version. On the other hand, the contribution of the family for the social participation stands out as significant but moderated. The nature of variables and the used scale can partially explain this result.

Key words: family cohesion, family adaptability, social participation, traumatic brain-injury, rehabilitation.

Service social - Volume 55, numéro 1, 2009 


\section{INTRODUCTION}

Les établissements de réadaptation en déficience physique de la province de Québec ont adopté le modèle du Processus de production du handicap comme cadre conceptuel de référence (Fougeyrollas, Cloutier, Bergeron, Côté, Côté et St-Michel, 1996). Ce modèle détermine l'apport de l'environnement social, tel que la famille, dans le processus de réadaptation et adopte de façon générale une perspective écologique en considérant l'interaction entre les facteurs personnels et environnementaux pour favoriser la participation sociale des personnes ayant des incapacités. Le modèle demeure toutefois imprécis quant à l'apport spécifique de la famille sur la participation sociale.

Une étude visant à déterminer les facteurs favorisant la participation sociale des adultes ayant subi un traumatisme craniocérébral (TCC) avait notamment pour objectif de documenter l'apport de l'environnement social (Dumont, Gervais, Fougeyrollas et Bertrand, 2004). Selon la consultation des études et écrits précédents, le soutien social est mentionné comme facteur favorable aux personnes ayant subi un TCC par de nombreux auteurs, et ce, à plusieurs niveaux de l'environnement social (Delgado, 1996; Levy et Wall, 2000; Nash et Bowen, 1999; Whiteneck, Fougeyrollas et Gerhart, 1997), soit le soutien familial (Calvert, 1997; Kelton, 2001; Kreutzer, Gervasio et Camplair, 1994; Meyerson, 2001; Wolkow et Ferguson, 2001), le soutien de la communauté ou les réseaux de soutien (Aitken et Morgan, 1999; Calvert, 1997; Delgado, 1996; Meyerson, 2001; Nash et Bowen, 1999; Wolkow et Ferguson, 2001), de même que le soutien en milieu de travail comme la cohésion entre les employés et le soutien des superviseurs (Aitken et Morgan, 1999). Le rôle des familles et des proches spécifiquement dans le processus de réadaptation des personnes ayant subi un traumatisme craniocérébral (TCC) est également reconnu (Gadoury, 1999; Ivy et Nicholson, 1999; McColl et al., 1999; Weiss et Puchta, 1999). De plus, certaines caractéristiques des familles et des milieux sont mentionnées comme étant favorables, telles que la cohésion et l'adaptabilité des familles, proposées dans le Circumplex Model élaboré par Olson et ses collègues (1983).

Dans le Circumplex Model, la cohésion est définie comme étant : « [...] the emotional bonding that family members have toward one another». (Traduction libre: les liens émotionnels qu'ont les membres de la famille entre eux). Quatre niveaux de cohésion sont définis : désengagé (très bas), séparé (bas à modéré), rapproché (modéré à élevé) et intriqué (très élevé). Les niveaux centraux de cohésion correspondent à un fonctionnement familial optimal. L’adaptabilité est définie comme étant : «[...] the ability of a marital or family system to change its power structure, role relationships, and relationship rules in response to situational and developmental stress ». (Traduction libre : l'habileté d'un système marital ou familial de changer sa structure de pouvoir, ses rôles relationnels et ses règles relationnelles en réponse au stress situationnel et développemental). Quatre niveaux d’adaptabilité sont définis : rigide (très bas), structuré (bas à modéré), flexible (modéré à élevé) et chaotique (très élevé). Les niveaux centraux d'adaptabilité correspondent à un fonctionnement familial optimal (Olson, Russell et Sprenkle, 1983). Relativement à ces deux caractéristiques, en plaçant une des dimensions en 
abscisse et l'autre en ordonnée, seize types de systèmes maritaux ou familiaux peuvent être identifiés (voir la figure 1).

L’instrument de mesure appelé Family Cohesion and Adaptability Evaluation Scale (FACES), qui est basé sur le Circumplex Model, était mentionné par McCubbin et Thompson (1987) comme pertinent et valide pour évaluer ces caractéristiques de la famille. Dans l'état actuel des connaissances, il n'a pas été démontré que ce modèle théorique s'applique à la population ayant subi un TCC (Olson, 1986; Olson, Bell et Portner, 1992). Toutefois, la version anglaise de cet outil a déjà été utilisée avec cette clientèle et a démontré sa capacité à discriminer différents types de familles dont un des membres a subi un TCC. Il pouvait donc s'avérer utile dans une étude visant à évaluer l'apport de la famille dans le processus de réadaptation (Perlesz, Kinsella et Crowe, 1999).

Figure 1

The Circumplex Model, traduction libre, tirée de: Olson, Russell et Sprenkle (1983)

$\longleftarrow$ Faible

Désengagé (D) Séparé (S) Rapproché (R) Intriqué (I)

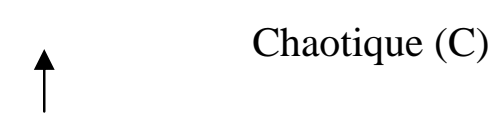

\section{Faible}

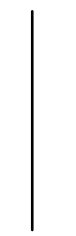

\section{Adaptabilité}

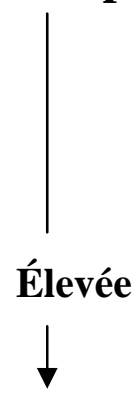

Rigide (Ri)

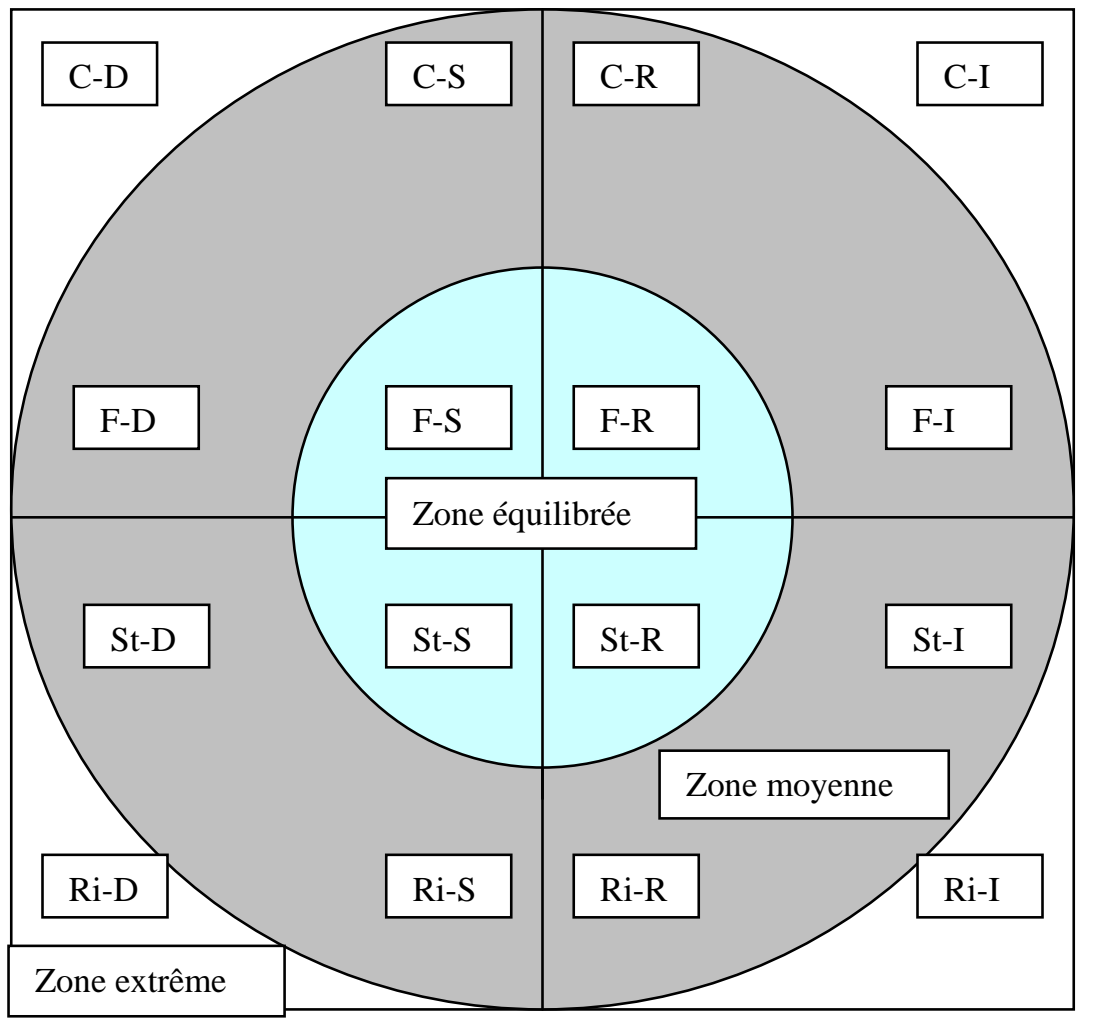


Le FACES mesure deux concepts relatifs aux familles ou aux couples, soit la cohésion et l'adaptabilité ou la capacité d'adaptation. Quatre versions du FACES ont été développées. La version II est celle qui a été retenue pour l'étude, car elle présente de meilleures qualités métrologiques que la version III et qu'elle est plus courte que la version I (Olson, Bell et Portner, 1992). Il est à noter que les étapes de validation de la version IV n’étaient pas terminées au moment de l'étude. Aucune version française validée de l’instrument n’était disponible.

Étant donné que le FACES II présentait les caractéristiques recherchées, mais n’était pas offert en français, l'étude poursuivait deux objectifs principaux :

1) obtenir une traduction française du FACES II ayant des propriétés métrologiques satisfaisantes sur le plan de la cohérence interne et de la validité du construit, en fonction des critères scientifiques généralement utilisés pour juger ces paramètres;

2) vérifier l'association entre les deux variables familiales (cohésion et adaptabilité) et la participation sociale des adultes ayant subi un TCC. Le degré d'association entre les variables constitue un indicateur de l'importance de l'apport de la famille pour la participation sociale des adultes ayant subi un TCC.

\section{Méthode}

\section{Objectif 1}

\section{L’instrument de mesure}

L’échelle de cohésion du FACES II comprend 16 énoncés relatifs aux concepts suivants : liens émotionnels, frontières de la famille, coalitions, temps, lieu, amis, prise de décision, intérêts et loisirs. L'échelle d'adaptabilité comprend 14 énoncés en relation avec les concepts suivants : revendication, leadership, discipline, négociation, rôles et règles. Les deux séries d'énoncés sont présentées en alternance dans un instrument unique comprenant 30 énoncés. Les réponses se distribuent selon une échelle de type Likert à 5 niveaux allant de presque jamais à presque toujours. Des exemples d'énoncés sont donnés à l’annexe 1.

Les trois premières versions anglaises du FACES ont démontré leur capacité à discriminer les familles ayant des difficultés (Olson, 1986). Elles ont également démontré une bonne validité et une bonne fidélité (McCubbin et Thompson, 1987; McCubbin, Thompson, Thompson et Fromer, 1998). Selon la documentation qui accompagne l'instrument, la version du FACES II retenue pour l'étude présente un indice alpha de Cronbach de 0,87 pour les énoncés évaluant la cohésion, de 0,78 pour les énoncés évaluant l'adaptabilité et de 0,90 pour l'échelle au total $(n=2412)$. La corrélation entre les deux échelles (cohésion et adaptabilité) s'avère modérée $(\mathrm{r}=0,25$ à 0,65$)$ et la corrélation avec la désirabilité sociale s'avère faible $(\mathrm{r}=0,38$ et 0,39$)$ (Olson, Bell et Portner, 1992). La stabilité a été examinée avec l'échelle de 50 énoncés (FACES I) et la corrélation de Pearson obtenue est de 0,84. La validité de critère concurrente 
avec le Dallas Self-Report Family Inventory est bonne, considérant une corrélation de 0,93 pour l'échelle de cohésion et de 0,79 pour l'échelle d'adaptabilité. De plus, les analyses factorielles font ressortir un construit dominant (Olson, Bell et Portner, 1992).

\section{Étapes}

La méthode suggérée par Vallerand (1989) a guidé l'ensemble de la démarche de traduction et de validation du FACES II. Les étapes comprenaient : 1) la préparation d'une version préliminaire par traduction renversée parallèle (Brislin, 1970); 2) l'évaluation de la version préliminaire par un comité formé de professionnels et de chercheurs; 3) prétest de la version expérimentale par méthode du sondage aléatoire auprès de cinq personnes volontaires ayant des incapacités; 4) consultation du comité en cas de difficultés; 5) analyse de la fidélité et de la validité par administration du test à un groupe de personnes volontaires ayant des incapacités.

\section{Procédure}

La traduction obtenue à la suite des étapes 1 à 4 a été administrée à un échantillon de personnes ayant des incapacités. Ces données permettaient de vérifier les propriétés métrologiques des instruments et de les comparer avec la version anglophone initiale. Les volontaires (étapes 3 et 5) ont été recrutés auprès des bénévoles de l’Institut de réadaptation en déficience physique de Québec (IRDPQ) et des usagers qui fréquentent des associations dont le siège social est situé dans les locaux de l'IRDPQ ou qui reçoivent des services à l'IRDPQ lors d'une de leurs visites. Les autorisations du comité d'éthique et de l'administration de l'IRDPQ ont été obtenues avant de procéder au recrutement des volontaires.

\section{Analyses}

Les propriétés métrologiques de la traduction ont été vérifiées, soit la cohérence interne (alpha de Cronbach) et la validité de construit (analyses factorielles). Les paramètres tirés de ces analyses ont été choisis en fonction des informations disponibles concernant le FACES II pour permettre la comparaison des versions anglaise et française (Olson, Bell et Portner, 1992). Le seuil retenu pour l'alpha de Cronbach est 0,8 pour que la cohérence interne soit jugée satisfaisante et d'autres paramètres reliés à ce type d'analyse ont également été vérifiés, comme la corrélation item totale corrigée (Streiner et Norman, 1995). L’analyse factorielle en composantes principales a été effectuée sans rotation et en analysant les deux premières composantes de la matrice, la première correspondant aux 16 énoncés relatifs à la cohésion (énoncés $1,3,5,7,9,11,13,15,17,19,21,23,25,27,29,30)$ et la seconde aux 14 énoncés relatifs à l'adaptabilité (énoncés 2, 4, 6, 8, 10, 12, 14, 16, 18, 20, 22, 24, 26, 28) (Olson, Bell et Portner, 1992). Les analyses ont été effectuées en regroupant l'échantillon de 27 personnes volontaires ayant des incapacités et un deuxième échantillon de 53 personnes ayant subi un TCC, soit l'échantillon associé au deuxième objectif de l'étude $(n=80)$. Ce regroupement permettait 
de se rapprocher des recommandations générales visant les analyses factorielles, soit 10 sujets par énoncé.

\section{Objectif 2}

Pour vérifier l'association entre la participation sociale et la cohésion ainsi que l'adaptabilité des familles, un instrument de mesure de la participation sociale et le FACES II ont été administrés à un échantillon de personnes adultes ayant subi un TCC. Il s’agissait d'une étude transversale corrélationnelle.

\section{Les critères de sélection des participants}

Les critères de sélection des participants sont les suivants : 1) avoir subi un TCC qui a nécessité une période de réadaptation intensive d'une durée variant de 2 semaines à 14 mois, ceci dans le but d'éliminer ceux qui n’ont pas de séquelles significatives (moins de 2 semaines de réadaptation intensive) et ceux qui ont de graves séquelles qui font qu'ils ne pourraient participer à l'étude (plus de 14 mois de réadaptation intensive); 2) avoir été admis à l'hôpital à la suite d'un traumatisme, puis avoir reçu des traitements dans les établissements de réadaptation désignés pour recevoir la clientèle TCC au Québec; 3) avoir reçu des services sur une base interne ou externe à l'IRDPQ; 4) avoir terminé la réadaptation intensive depuis plus d'un an et moins de cinq ans, soit entre l'été 1996 et l'été 2001; 5) demeurer dans un périmètre de 100 kilomètres de la ville de Québec; 6) être âgés de 16 à 50 ans au moment du traumatisme; 7) avoir au moins 18 ans au moment de participer à l'étude; 8) être francophone; 9) avoir la capacité d'accomplir les tâches reliées à la collecte de données. Une incapacité sur ce plan peut être causée par des problèmes physiques, telle l'incapacité à lire, ou des incapacités psychologiques, telle une dépression majeure. Un critère d'exclusion est aussi appliqué, soit de ne pas avoir de conditions associées majeures nécessitant l'admission dans un autre programme de réadaptation de l'IRDPQ, comme une lésion médullaire. En effet, la personne présente un profil clinique différent à ce moment sur de nombreux plans, ce qui induit des biais, nuit à l'interprétation des résultats et à la formulation de recommandations pour la clientèle visée.

\section{L’instrument de mesure}

La mesure de la participation sociale a été réalisée avec la Mesure des habitudes de vie (MHAVIE) (Noreau et Fougeyrollas, 1996). Les qualités de cet instrument sont reconnues dans le domaine social pour l'évaluation des personnes ayant des incapacités (Dijkers, Whiteneck et El-Jaroudi, 2000) et il a déjà été utilisé avec certaines clientèles, tel que les personnes ayant subi un traumatisme à la moelle épinière ou un TCC (Noreau et Fougeyrollas, 2000). La MHAVIE comprend 69 énoncés pouvant être regroupés en 12 catégories : alimentation, état général, soins personnels, communication, activités domestiques, déplacements, responsabilités, relations interpersonnelles, consommation de biens et services, éducation, travail et loisirs. Des exemples d'énoncés sont donnés à l'annexe 2. Pour chaque énoncé, le répondant doit d'abord indiquer le niveau de réalisation sur une échelle à 4 points (sans difficulté à dépendance totale) ainsi que le 
type d'aide requis (aucune, aide technique, aménagement, aide humaine). Il doit finalement indiquer le niveau de satisfaction sur une échelle à 5 points (de très insatisfait à très satisfait). Dans le cadre de cette étude, la réponse de chaque énoncé est ramenée sur une échelle de type Likert à 4 niveaux : 4) réalise l'habitude de vie sans difficulté, avec ou sans aide technique; 3) réalise l'habitude de vie avec difficulté, mais sans aide humaine, avec ou sans aide technique; 2) réalise l'habitude de vie avec aide humaine, avec ou sans aide technique; et 1) incapable de réaliser l'habitude de vie ou dépendance totale. Le choix de cette échelle en 4 points s’appuie sur des études antérieures ayant utilisé cet instrument auprès de cette clientèle. Cette façon de faire assure en effet une meilleure qualité de mesure, car la répartition des réponses est plus uniforme dans les catégories et qu'il n’y a pas de catégories avec très peu ou pas de réponses (Dumont, Bertrand, Fougeyrollas et Gervais, 2003; Dumont, Gervais, Fougeyrollas et Bertrand, 2004). Il faut également mentionner que l'échelle de satisfaction n'est pas utilisée étant donné que cette variable (la satisfaction) n'a pas été retenue pour l'étude. Les résultats de chaque individu sont calculés suivant le modèle de mesure classique, soit l'addition du score obtenu à chaque énoncé pour obtenir le score global de participation sociale (Dumont, Gervais, Fougeyrollas et Bertrand, 2004).

La validité de cet instrument a été démontrée par de nombreuses consultations et par le consensus établi au Québec autour du modèle conceptuel à la base de l'instrument, soit le modèle du Processus de production du handicap (Fougeyrollas, 1997; Fougeyrollas, Cloutier, Bergeron, Côté, Côté et St-Michel, 1996). L’instrument a été validé auprès de plusieurs clientèles incluant les personnes ayant subi un TCC. Sa fidélité test-retest intraexaminateur a été démontrée auprès de deux échantillons, soit un échantillon de 28 personnes avec déficiences motrices et un autre de 30 adultes et 22 enfants avec déficiences motrices associées à des déficiences cognitives. La fidélité interexaminateurs a, par ailleurs, été testée auprès d’un échantillon de 20 adultes ayant des déficiences motrices associées à des déficiences cognitives ou du langage. La valeur du coefficient de corrélation intraclasse obtenu est de 0,83 , ce qui est considéré comme étant satisfaisant (Fougeyrollas et al., 1998).

\section{Variables et analyses}

Les résultats descriptifs à l'instrument de mesure FACES II ont été calculés suivant les consignes des auteurs (Olson, Bell et Portner, 1992). La corrélation ainsi que la régression simple et multiple ont été utilisées pour vérifier les associations entre les variables. La régression multiple a été faite selon le mode entrée. La variable dépendante est la participation sociale, les variables indépendantes sont la cohésion et l’adaptabilité des couples ou des familles.

\section{Procédure}

Les participants ont été recrutés parmi les usagers ayant reçu des services à l'IRDPQ. Préalablement au recrutement et à la collecte de données, les autorisations de la part du comité d'éthique et de la direction de l'IRDPQ ont été obtenues. Le recrutement s'est déroulé en trois étapes : 
1) La population a été constituée à partir du fichier des archives de l'IRDPQ qui contient la liste des personnes ayant reçu des services de réadaptation. Du groupe de personnes répondant aux critères de sélection, un échantillon aléatoire de sept groupes de 50 personnes a été constitué.

2) Une lettre a été envoyée au premier groupe de 50 personnes pour leur expliquer brièvement l'étude et la nature de la participation attendue. Ils signifiaient leur accord à participer à l'étude par une lettre qu'ils signaient et retournaient par le courrier dans une enveloppe préadressée et affranchie.

3) Ceux qui ont accepté ont été rejoints par téléphone pour fixer un rendez-vous.

Quelques semaines après le premier envoi, un autre envoi de 50 lettres était effectué. La procédure se répétait ainsi jusqu’à ce que toutes les personnes répondant aux critères de sélection aient été sollicitées.

La MHAVIE était remplie par un examinateur expérimenté lors d’une entrevue individuelle. Les participants remplissaient eux-mêmes le FACES II lors de la même rencontre.

\section{Résultats}

\section{Objectif 1}

Concernant les analyses de cohérence interne du FACES II, l'alpha de Cronbach obtenu pour l'échelle totale est de 0,90 , il est de 0,89 pour l'échelle de cohésion (16 énoncés) et de 0,74 pour l'échelle d'adaptabilité (14 énoncés) $(n=76)$. Quatre sujets tirés du premier échantillon de 27 personnes ont été retirés de ces analyses, car ils n’avaient pas répondu à tous les énoncés. Pour l'échelle totale, un seul énoncé corrèle de façon négative (corrélation item-total corrigée) avec l'ensemble des énoncés, soit l'énoncé 12. L’énoncé 24 présente une faible corrélation itemtotal corrigée soit 0,005 . En ce qui concerne les analyses de chaque échelle séparément, aucun énoncé ne corrèle de façon négative avec l'échelle de cohésion, la plus faible corrélation itemtotal corrigée étant de 0,235. Les énoncés 12 et 24 ont respectivement une corrélation item-total corrigée négative $(-0,199)$ ou une faible corrélation item-total corrigée $(0,010)$ avec l'échelle d'adaptabilité. L'alpha monte à 0,78 si on élimine l'énoncé 12 de l'échelle d'adaptabilité et à 0,76 si on élimine l’énoncé 24 .

Les résultats des analyses factorielles font ressortir 9 composantes qui expliquent $70 \%$ de la variance (Eigenvalues $>1$ ), la première composante expliquant $31 \%$ de la variance $($ Eigenvalue $=9,4)$. Les deux premières composantes de la matrice des composantes tirée de l'analyse en composantes principales sont présentées au tableau 1 (analyse factorielle de l'échelle totale). Tous les énoncés de l'échelle de cohésion sont associés positivement à la première composante. Par contre, certains énoncés de l'échelle d'adaptabilité ne sont pas en relation inverse avec la seconde composante, soit les énoncés 12, 22 et 24, contrairement aux autres énoncés de cette échelle. 
Tableau 1

Analyse des composantes principales : valeur des saturations des composantes 1 et 2 tirées des données publiées et des analyses effectuées

\begin{tabular}{|c|c|c|c|c|}
\hline \multirow[b]{2}{*}{ Énoncé } & \multicolumn{2}{|c|}{$\begin{array}{l}\text { Version anglaise } \\
\quad(n=2412)\end{array}$} & \multicolumn{2}{|c|}{$\begin{array}{l}\text { Traduction française } \\
\qquad(\mathbf{n}=\mathbf{8 0})\end{array}$} \\
\hline & Composante 1 & Composante 2 & Composante 1 & Composante 2 \\
\hline 1 & 0,47 & & 0,54 & \\
\hline 2 & & 0,52 & & $-0,32$ \\
\hline 3 & 0,54 & & 0,54 & \\
\hline 4 & & 0,55 & & $-0,06$ \\
\hline 5 & 0,47 & & 0,62 & \\
\hline 6 & & 0,49 & & $-0,29$ \\
\hline 7 & 0,61 & & 0,84 & \\
\hline 8 & & 0,48 & & $-0,14$ \\
\hline 9 & 0,58 & & 0,25 & \\
\hline 10 & & 0,24 & & $-0,18$ \\
\hline 11 & 0,35 & & 0,39 & \\
\hline 12 & & 0,10 & & 0,11 \\
\hline 13 & 0,34 & & 0,62 & \\
\hline 14 & & 0,39 & & $-0,58$ \\
\hline 15 & 0,48 & & 0,61 & \\
\hline 16 & & 0,46 & & $-0,24$ \\
\hline 17 & 0,56 & & 0,82 & \\
\hline 18 & & 0,22 & & $-0,29$ \\
\hline 19 & 0,58 & & 0,69 & \\
\hline 20 & & 0,44 & & $-0,23$ \\
\hline 21 & 0,47 & & 0,68 & \\
\hline 22 & & 0,39 & & 0,14 \\
\hline 23 & 0,61 & & 0,76 & \\
\hline 24 & & 0,19 & & 0,30 \\
\hline 25 & 0,51 & & 0,36 & \\
\hline 26 & & 0,49 & & $-0,04$ \\
\hline 27 & 0,35 & & 0,39 & \\
\hline 28 & & 0,26 & & $-0,14$ \\
\hline 29 & 0,43 & & 0,61 & \\
\hline 30 & 0,51 & & 0,79 & \\
\hline
\end{tabular}

\section{Objectif 2}

Les résultats descriptifs concernant les échelles de cohésion et d'adaptabilité des couples et des familles sont présentés au tableau 2. En fonction des critères définis par les auteurs, l'ensemble de l'échantillon se situe dans la catégorie modérément équilibrée. La valeur de la cohésion obtenue est très voisine à la valeur centrale du modèle (la valeur limite est de 60), soit à cheval sur les catégories " rapproché » et « séparé » par opposition aux catégories " désengagé » et «intriqué ». Sur le plan de l’adaptabilité, les résultats se situent dans la partie moyenne supérieure de la catégorie « flexible », par opposition aux catégories « chaotique », "structuré » 
et « rigide ». Parmi les seize types de couples et de familles proposés dans le Circumplex Model, les répondants de l'étude se situent donc à la limite des catégories « rapproché flexible » et " séparé flexible ».

Sur le plan de l'association entre les variables, on note que les échelles de cohésion et d'adaptabilité corrèlent fortement entre elles (voir le tableau 3), mais que la corrélation avec la participation sociale est modérée. Les analyses de régression simple et multiple démontrent un taux d'explication de la participation sociale avec ces deux variables familiales (voir le tableau 4) légèrement plus important pour la cohésion que pour l’adaptabilité des familles.

Tableau 2

Résultats à l'échelle de cohésion et adaptabilité des couples et des familles $(n=53)$

\begin{tabular}{|l|c|c|}
\hline \multicolumn{1}{|c|}{ Échelle } & Moyenne (écart-type) & Étendue de la catégorie \\
\hline Cohésion & $60,21(10,6)$ & Rapproché $: 60$ à 70 \\
Adaptabilité & $50,47(6,6)$ & Flexible : 46 à 54 \\
\hline
\end{tabular}

Tableau 3

Matrice de corrélations entre les résultats obtenus aux instruments mesurant les variables à l'étude $(n=53)$

\begin{tabular}{|l|c|c|c|}
\hline & Cohésion. & Adaptabilité & Participation sociale \\
\hline Cohésion & 1 & & \\
\hline Adaptabilité & $0,74^{*}$ & 1 & \\
\hline Participation sociale & $0,38^{* *}$ & $0,36^{* * *}$ & 1 \\
\hline
\end{tabular}

$* \mathrm{p}<0,0001$

$* * \mathrm{p}=0,005$

$* * * \mathrm{p}=0,008$ 
Tableau 4

Paramètres permettant de déterminer si les variables reliées à la famille expliquent la participation sociale (régression simple et régression multiple selon le mode entrée) $(n=53)$

\begin{tabular}{|l|c|c|}
\hline \multicolumn{1}{|c|}{ Variable (s) } & $\begin{array}{c}\text { Coefficient de détermination (R2) et } \\
\text { valeur p }\end{array}$ & $\begin{array}{c}\text { Bêta } \\
\text { (non standardisé) }\end{array}$ \\
\hline Adaptabilité & $0,131(\mathrm{p}=0,008)$ & 1,051 \\
Cohésion & $0,147(\mathrm{p}=0,005)$ & 0,699 \\
Adaptabilité + cohésion & $0,161(\mathrm{p}=0,012)$ & Cohésion : 0,469 \\
(mode entrée) & & Adaptabilité : 0,499 \\
\hline
\end{tabular}

\section{DISCUSSION ET CONCLUSION}

L’étude visait deux objectifs. En ce qui concerne l'obtention d'une version francophone du FACES II ayant de bonnes propriétés métrologiques, les analyses effectuées démontrent que l'instrument se comporte comme la version anglophone et relativement peu de différences sont notées. En effet, les résultats publiés par les auteurs du test sur les analyses de cohérence interne (alpha de Cronbach) sont respectivement de 0,90, 0,87 et 0,78 ( $\mathrm{n}=2412$ ) (Olson, Bell et Portner, 1992), ce qui est très proche des résultats ici obtenus $(0,90,0,89$ et 0,74$)$.

Selon les résultats des analyses factorielles, les énoncés 12 et 24 se comportent différemment dans la matrice des composantes. Ces tendances sont aussi observées dans la version anglaise de l'instrument, ces deux énoncés ayant les plus faibles valeurs de composantes $(0,10$ et 0,19$)$. De plus, leur corrélation item-total corrigée était aussi faible ou négative dans les analyses de cohérence interne. Ces deux énoncés font partie de l'échelle d'adaptabilité et portent sur les règles dans la famille ou le couple. Le premier stipule que les règles sont difficiles à connaître et le second que les règles sont difficiles à changer. La notion de "règle » serait ainsi moins associée au concept d'adaptabilité selon les résultats obtenus.

Considérant l'ensemble des résultats obtenus, certains écarts peuvent également être expliqués par le fait que la famille québécoise vit des bouleversements importants en comparaison avec la famille traditionnelle, ce qui modifie la structure des réponses. En effet, les réponses peuvent être différentes en fonction des valeurs sociales et des contextes, notamment les énoncés concernant les règles.

Globalement, les particularités de l'échantillon à l'étude et le relativement petit nombre de sujets peuvent expliquer les différences observées avec les résultats des analyses de la version 
anglophone du test. Aucune modification à la traduction n’est recommandée à la lumière de ces résultats.

En ce qui concerne le deuxième objectif, la cohésion et l'adaptabilité des familles expliquent une proportion significative quoique relativement modeste de la participation sociale des personnes ayant subi un TCC. Un tel résultat est attendu, car de nombreux autres facteurs peuvent expliquer la participation sociale, notamment le degré de sévérité du TCC, la volonté et autre (Dumont, Gervais, Fougeyrollas et Bertrand, 2004). Il est également possible que l'apport de la famille ne se fasse pas sur le plan de la participation sociale des personnes, mais agisse sur un autre plan : soutien émotif, soutien pendant la phase de réadaptation intensive et non à l'étape où se situaient les personnes après la fin des services intensifs de réadaptation. Il est également possible que les deux caractéristiques mesurées (cohésion et adaptabilité) ne soient pas celles qui vont avoir le plus d'influence sur la participation sociale, même si elles peuvent influencer d'autres aspects comme l'estime de soi ou les relations interpersonnelles. Enfin, l'instrument peut présenter certaines limites en fonction de la nature des variables mesurées. En effet, selon le modèle théorique à la base de l'instrument, une cohésion ou adaptabilité trop grande est aussi néfaste que si elle est trop faible. Les résultats faibles et élevés à cette échelle démontrent des situations problématiques, tandis que les résultats moyens sont ceux qui indiquent la situation la plus favorable. Pour la participation sociale, plus le résultat est élevé, plus la participation sociale est élevée, ce qui est l'objectif souhaité. La corrélation ou les analyses de régression entre ces deux instruments peuvent donc présenter des difficultés d'interprétation, car il est attendu sur le plan théorique qu'un score moyen au FACES II sera associé à un score élevé de participation sociale. Par ailleurs, la corrélation entre les échelles de cohésion et d'adaptabilité est plus élevée $(\mathrm{r}=0,74)$ que la valeur rapportée par les auteurs dans leur document de référence $(\mathrm{r}=0,25$ à 0,65) (Olson, Bell et Portner, 1992). Ces deux concepts sont donc ici liés. Les analyses de régression pourraient donc être réalisées avec le résultat global (échelles de cohésion et adaptabilité réunies) dans certaines situations.

La recommandation finale est donc qu'il est possible d'utiliser la version francophone du FACES II telle que traduite, mais que l'échelle de mesure pourrait être modifiée pour être utilisée dans les études corrélationnelles visant à vérifier l'association avec une autre variable, le résultat moyen (actuellement au score de 3) devant correspondre au chiffre le plus élevé (5), car il s’agit de la situation recherchée. 


\section{BiBLIOGRAPHIE}

AitKen, S., et J. Morgan (1999). « How Motorola promotes good health », The Journal for Quality et Participation, vol. 22, $\mathrm{n}^{\mathrm{0}}$ 1, p. 54-57.

BRISLIN, R.W. (1970). « Back translation for cross-cultural research », Journal of Cross-Cultural Psychology, $\mathrm{n}^{\mathrm{0}}$ 1, p. 185-216.

CAlvert, W.J. (1997). "Protective factors within the family, and their role in fostering resiliency in African American adolescents », Journal of Culture Diversity, vol. 4, n ${ }^{0}$, p. $110-117$.

Delgado, M. (1996). «Implementing a Natural Support System AOD Project: Administrative considerations and recommendations ", Alcoholism Treatment Quarterly, vol. 14, $\mathrm{n}^{0}$ 2, p. 1-14.

Dumont, C., R. Bertrand, P. Fougeyrollas, et M. Gervais (2003). « Rasch modeling and the measurement of social participation ", Journal of Applied Measurement, vol. 4, $n^{0} 4$, p. 309-325.

Dumont, C., M. Gervais, P. Fougeyrollas, et R. Bertrand (2004). « Toward an explanatory model of social participation for adults with traumatic brain injury », Journal of Head Trauma and Rehabilitation, vol. 19, n ${ }^{\circ}$ 6, p. 431-444.

DiJKers, M., G. WhitenECK, et R. EL-JAROudi (2000). « Tools of disability outcomes research. Measures of social outcomes in disability research ", Archives of Physical Medicine and Rehabilitation, $\mathrm{n}^{0}$ 8, Suppl. 2, p. S63-S80.

Fougeyrollas, P. (1997). « Les déterminants environnementaux de la participation sociale des personnes ayant des incapacités : le défi socio-politique de la révision de la CIDIH », Revue Canadienne de réadaptation, vol. 10, n² 2, p. 147-160.

Fougeyrollas, P., R. Cloutier, H. Bergeron, J. Côté, M. Côté, et G. St-Michel, (1996). Révision de la proposition québécoise de classification: Processus de production du handicap, Québec, Réseau international sur le processus de production du handicap, 136 p.

Fougeyrollas, P., et al. (1998). "Social consequences of long term impairments and disabilities: Conceptual approach and assessment of handicap », International Journal of Rehabilitation Research, vol. 21, n 2, p. 127-141.

GADOURY, M. (1999). Cadre de référence clinique pour l'élaboration de programmes de réadaptation pour la clientèle qui a subi un traumatisme craniocérébral, Volet adulte, Direction des politiques et programmes pour les accidentés, Service de la programmation en réadaptation, Société de l'assurance automobile du Québec, 156 p.

IVY, N., et D.E. NiCHOLSON (1999). « Prognostic and socioeconomic variables in rehabilitation admission decisions for patients with traumatic brain injury », Neurology Report, vol. 23, $n^{0} 2$, p. 68-73. 
Kelton, R.W. (2001). « Facing up to stigma: Workplace and personal strategies », Cleft Palate Craniofacial Journal, vol. 38, n⿳0 3, p. 245-247.

Kreutzer, J.S., A.H. Gervasio, et P.S. CAMPlair (1994). « Primary caregivers’ psychological status and family functioning after traumatic brain injury », Brain Injury, vol. 8, $\mathrm{n}^{0}$ 3, p. 197-210.

LEVY, A.J., et J.C. Wall (2000). «Children who have witnessed community homicide: Incorporating risk and resilience in clinical work », Families in Society: The Journal of Contemporary Human Services, vol. 81, n 4, p. 402-411.

MCColl, M.A., et al. (1999). «Transitions to independent living after ABI », Brain Injury, vol. 13, n ${ }^{0} 5$, p. 311-330.

McCubBin, H.I., et A.I. ThOMPson (dir.) (1987). Family assessment inventories for research and practice, Madison, University of Wisconsin-Madison, $343 \mathrm{p}$.

McCubbin, H.I., E.A. Thompson, A.I. Thompson, et J.E. Fromer (dir.) (1998). Stress, Coping and Health in Families: Sense of Coherence and Resiliency, Thousand Oaks, Sage, 313 p.

Meyerson, M.D. (2001). "Resiliency and success in adults with Moebius syndrome », Cleft Palate Craniofacial Journal, vol. 38, n 3, p. 231-235.

NASH, J.K., et G.L. Bowen (1999). "Perceived crime and informal social control in the neighbourhood as a context for adolescent behavior: A risk for resilience perspective ", Social Work Research, vol. 23, n 3, p. 171-186.

Noreau, L., et P. Fougeyrollas (1996). « L’évaluation des situations de handicap : la 'Mesure des habitudes de vie’ appliquée aux personnes ayant une lésion à la moelle épinière », Revue Canadienne de réadaptation, vol. 10, $\mathrm{n}^{0}$ 2, p. 81-97.

Noreau, L., et P. Fougeyrollas (2000). « Long-term consequences of spinal cord injury on social participation: the occurrence of handicap situations », Disability and Rehabilitation, vol. 22, $\mathrm{n}^{\mathrm{0}}$ 4, p. 170-180.

Olson, D.H., C.S. Russell, et D.H. Sprenkle (1983). «Circumplex Model of marital and family system: VI. Theoretical update », Family Process, n 22, p. 69-83.

Olson, D.H. (1986). «Circumplex Model VII: Validation studies and FACES III », Family Process, $\mathrm{n}^{0}$ 25, p. 337-351.

Olson, D.H., R. Bell, et J. Portner (1992). «FACES II », Family Inventories Manual, Minneapolis, Life Innovations.

Perlesz, A., G. Kinsella, et S. Crowe (1999). «Impact of traumatic brain injury on the family: a critical review », Rehabilitation Psychology, vol. 44, n 1, p. 6-35.

StreineR, D.L., et G.R. Norman (1995). Health Measurement Scales: a practical guide to their development and use, Oxford, Oxford University Press. 
VALlERAND, R.J. (1989). «Vers une méthodologie de validation transculturelle de questionnaires psychologiques : implications pour la recherche en langue française », Psychologie Canadienne, n ${ }^{0}$ 30, p. 662-680.

WEISS, D.M., et J.B. PuCHTA (1999). «Independent living center services for post-hospital traumatic brain injury patients », The Journal of Cognitive Rehabilitation, vol. 17, $\mathrm{n}^{0}$ 6, p. 6-12.

Whiteneck, G.G., P. Fougeyrollas, et K.A. Gerhart (1997). « Elaborating the Model of Disablement » dans : M.J. FuHRER (dir.), Assessing Medical Rehabilitation Practices. The Promise of Outcomes Research, Baltimore, MD, Paul H. Brookes Publishing Co.

Wolkow, K.E., et H.B. Ferguson (2001). "Community factors in the development of resiliency: considerations and future directions ", Community Mental Health Journal, vol. $37, n^{\circ} 6$, p. $489-498$. 


\section{ANNEXE 1}

\section{Exemples d'énoncés du FACES II}

- Chaque membre de la famille a son mot à dire en regard des décisions familiales majeures.

- Dans notre famille, chacun fait à sa guise.

- Les membres de la famille se sentent plus proches de personnes extérieures à la famille que des membres de la famille.

- $\quad$ Il est difficile de faire changer une règle dans notre famille.

- Les membres de la famille partagent des intérêts et des passe-temps entre eux.

\section{ANNEXE 2.}

\section{Exemples d'énoncés de la Mesure des habitudes de vie}

- Préparer vos repas (incluant l’utilisation des appareils électroménagers)

- Vous habiller et vous déshabiller le haut du corps (vêtements, accessoires, incluant le choix des vêtements)

- Communiquer par écrit (écrire une lettre, un message...)

- Utiliser des moyens de transport comme conducteur

- Maintenir des relations affectives avec vos enfants

- Utiliser les services publics de votre milieu (santé, gouvernementaux, bancaires, juridiques ...)

- Participer à des organisations sociocommunautaires (clubs sociaux, groupe d'entraide ou religieux...)

- Participer à des activités scolaires ou de formation professionnelle de niveau

- Occuper un travail rémunéré

- Pratiquer des activités artistiques, culturelles ou artisanales (musique, danse, menuiserie...) 